\title{
Dual-plane stereo particle image velocimetry (DSPIV) for measuring velocity gradient fields at intermediate and small scales of turbulent flows
}

\author{
John A. Mullin, Werner J. A. Dahm
}

\begin{abstract}
A two-frequency dual-plane stereo particle image velocimetry (DSPIV) technique is described for highly resolved measurements of the complete nine-component velocity gradient tensor field $\partial u_{i} / \partial x_{j}$ on the quasi-universal intermediate and small scales of turbulent flows. The method is based on two simultaneous, independent stereo particle image velocimetry (PIV) measurements in two differentially spaced light sheet planes, with light sheet characterization measurements demonstrating the required sheet thicknesses, separation, and two-axis parallelism that determine the measurement resolution and accuracy. The present approach uses an asymmetric forward-forward scatter configuration with two different laser frequencies in conjunction with filters to separate the scattered light onto the individual stereo camera pairs, allowing solid metal oxide particles to be used as seed particles to permit measurements in nonreacting as well as exothermic reacting turbulent flows.
\end{abstract}

\section{1}

\section{Introduction}

Much of the experimental research in turbulence is aimed at developing models for the quasi-universal intermediate and small scales of both nonreacting and highly exothermic reacting turbulent flows. This requires information on the structure and dynamics at these scales in various gradient fields, such as the strain rate and vorticity fields and the kinetic energy dissipation rate field, obtained from the velocity derivatives $\partial u_{i} / \partial x_{j}$. Owing to the difficulty in experimentally measuring all nine simultaneous components of these velocity gradients at the intermediate and small scales of turbulent flows, these gradient fields have, to date, been studied primarily by direct numerical simulations of comparatively simple turbulent flows.

Experimental measurements of velocity gradients in turbulent flows originated with Batchelor and Townsend

Received: 18 February 2004 / Revised: 24 September 2004

Accepted: 7 October 2004

Published online: 22 December 2004

(C) Springer-Verlag 2004

\section{J. A. Mullin, W. J. A. Dahm ( $\square)$}

Laboratory for Turbulence and Combustion (LTC),

Department of Aerospace Engineering,

The University of Michigan,

Ann Arbor MI, 48109-2140, USA

E-mail: wdahm@umich.edu
(1949), who used single-point time-series data from hotwire measurements with Taylor's hypothesis to approximate a single component of the velocity gradient tensor. Numerous subsequent studies have developed increasingly capable multiple-wire probes to simultaneously measure several components of the velocity gradient tensor (e.g., Kovasznay 1950; Kistler 1952; Foss 1976; Antonia et al. 1998). A few studies have used probes with up to 20 hotwires to measure all nine components of the velocity gradient tensor at a single point (Antonia et al. 1987; Brown et al. 1987; Balint et al. 1989; Tsinober et al. 1992; Kit et al. 1993).

Particle image velocimetry (PIV) allows the simultaneous nonintrusive measurement of two in-plane velocity components, here denoted $u(x, y)$ and $v(x, y)$. These provide four of the nine velocity gradient tensor components $\partial u_{i} / \partial x_{j}$, which, in turn, give access to three of the six components of the strain rate tensor and a single vorticity component. Stereo PIV, dual-plane PIV (Raffel et al. 1998), and scanning PIV (Brückner 1997) additionally provide the out-of-plane velocity component $w(x, y)$ and, thereby, provide two further velocity gradient components $\partial w / \partial x$ and $\partial w / \partial y$. However, these do not give access to any additional components of either the strain rate tensor or the vorticity vector.

Particle tracking velocimetry (PTV) provides threecomponent velocity fields throughout a three-dimensional volume (e.g., Kasagi and Nishino 1991; Malik et al. 1993). However, the comparatively low spatial resolution imposed by the large particle separations needed to allow accurate particle tracking prevents velocity gradient measurements at comparatively small scales of turbulence. The most extensive velocity gradient measurements in turbulent flows to date have come from holographic particle image velocimetry (HPIV) (Zhang et al. 1997), though the resolution in those measurements is significantly larger than the smallest scales in the turbulent flow.

Indirect measurements of $\partial u_{i} / \partial x_{j}$ via scalar imaging velocimetry (SIV) are based on three-dimensional laserinduced fluorescence imaging of a scalar field and inversion of the conserved scalar transport equation from the measured scalar field data to obtain the underlying three-component velocity field. This has allowed the first noninvasive measurements of all nine simultaneous components of the velocity gradients at the intermediate and small scales of a turbulent flow (Dahm et al. 1992; Su and Dahm 1996a, 1996b). However, the results are obtained indirectly from measured scalar field data and require additional smoothness and continuity constraints in the inversion to obtain the velocity field data. 
A dual-plane stereo particle image velocimetry (DSPIV) measurement was first reported by Kähler and Kompenhans (1999), in which two separate stereo PIV systems were used to provide all three components of velocity in two parallel light sheets. The method required separating the light scattered by particles in the two light sheets onto two independent stereo camera pairs. That study used a polarization-based approach, in which the two light sheets were arranged with orthogonal polarizations so that each stereo camera pair saw the scattered light from only one of the sheets. Such an approach allows all nine components of the velocity gradients to be determined from the measured velocities in the two parallel light-sheet planes.

Kähler et al. (2002) used this to measure comparatively large-scale features of the flow in a turbulent boundary layer, and $\mathrm{Hu}$ et al. (2001) used the same technique to investigate large-scale features of a lobed jet mixer. Neither of these studies attempted to resolve the velocity gradients on the quasi-universal intermediate and small scales of turbulent flows. Moreover, maintaining the orthogonal polarization in the Mie scattered light required the scattering particles to be spherical, and as a consequence, these studies used fine liquid droplets as the seed particles. This can be done in nonreacting turbulent flows, but in exothermic reacting flows, such liquid droplets do not survive and the polarization-based method cannot be used.

The two-frequency DSPIV technique presented here allows direct noninvasive measurements of all nine components of the velocity gradient tensor field $\partial u_{i} / \partial x_{j}$ at the intermediate and small scales of turbulent flows. As indicated in Fig. 1, the technique is based on DSPIV measurements using two different laser frequencies in conjunction with filters to separate the light scattered from

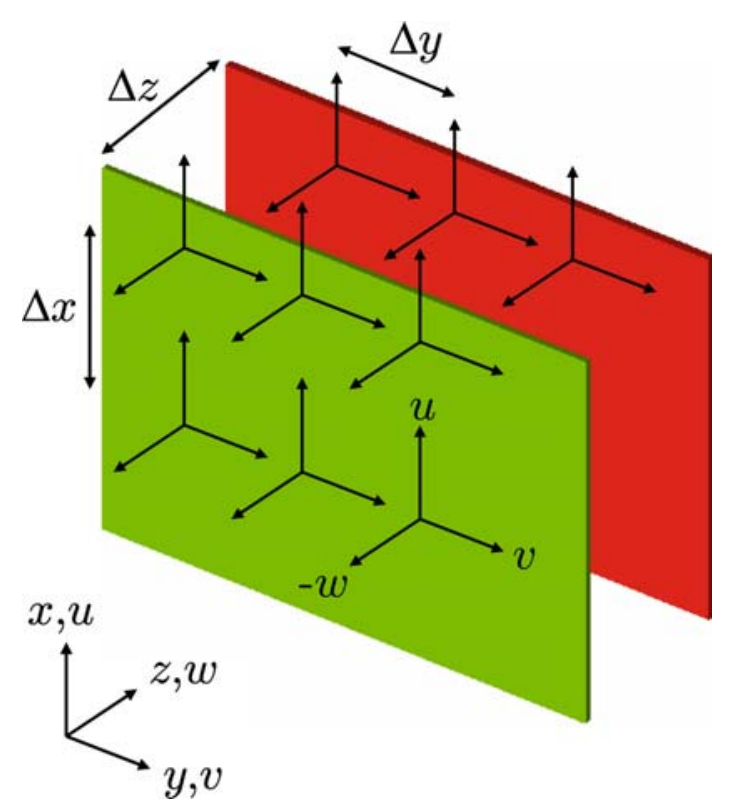

Fig. 1. Basic principle for frequency-based dual-plane stereo PIV (DSPIV) measurements, consisting of two simultaneous independent stereo PIV measurements of all three velocity components $(u, v, w)$ in two differentially spaced parallel light sheets, one at $532 \mathrm{~nm}$ (green) and one at $635 \mathrm{~nm}$ (red) the seed particles onto the individual stereo camera pairs. This allows traditional solid metal oxide particles to be used as the seed, and, thereby, permits such two-frequency DSPIV measurements to be made in reacting as well as nonreacting flows. Moreover, whereas both the dual-plane measurements of Kähler and Kompenhans (1999) and $\mathrm{Hu}$ et al. (2001) used a single-axis forward-backward imaging configuration, the present measurements are based on a two-axis forward-forward stereo camera configuration that allows accurate measurements at lower laser sheet energies. Furthermore, Kähler and Kompenhans (1999) used an included angle of $90^{\circ}$ that minimized the out-ofplane velocity component error, and $\mathrm{Hu}$ et al. (2001) used a symmetric arrangement with a $50^{\circ}$ included angle that minimized the ratio of out-of-plane to in-plane velocity component errors. By contrast, the present approach uses an asymmetric camera arrangement that achieves the same $50^{\circ}$ included angle in a forward-forward camera configuration, providing the benefits of forward imaging while still minimizing the ratio of out-of-plane to in-plane velocity component errors. Finally, the present measurement system provides for significantly higher spatial resolution than has been achieved in any prior DSPIV measurement. Specifically, the spatial resolution achieved here is a factor of 3-4 times finer than the local inner (viscous) length scale $\lambda_{v}$ of the turbulent flow; in comparison, the earlier measurements of $\mathrm{Hu}$ et al. (2001) correspond to a relative resolution at least a factor of 20 times coarser than this system at the furthest downstream location, where the resolution was highest.

Here, we describe the key components of this two-frequency DSPIV measurement approach, including a detailed discussion of the camera configuration, the dual-plane light sheet positioning and quantitative characterization, and the resulting associated errors inherent in this DSPIV technique. We also demonstrate the successful realization and characterization of such a system to obtain simultaneous measurements of all nine components of the velocity gradient tensor field $\partial u_{i} / \partial x_{j}$ resolved to the smallest scales of a turbulent shear flow. Whereas the present paper focused on the measurement technique itself, two companion papers (Mullin and Dahm 2004a, 2004b) provide additional extensive results from the application of this two-frequency DSPIV technique in detailed studies of the structure, statistics, similarity, and scaling of velocity gradient fields, strain rate and vorticity fields, and kinetic energy dissipation rate fields on the quasi-universal intermediate and small scales of a turbulent shear flow.

2

\section{Two-frequency DSPIV system configuration}

The present two-frequency DSPIV system, shown in Fig. 2, consists primarily of four Nd:YAG lasers, two pulsed dye lasers, and four charge-coupled device (CCD) cameras coordinated by a computer with an onboard programmable timing unit. It is based on two, essentially independent, stereo PIV systems that simultaneously provide measurements in two differentially spaced data planes. Each stereo camera pair operates with a coincident pair of $532-\mathrm{nm}$ or $635-\mathrm{nm}$ laser light sheets that illuminate the 


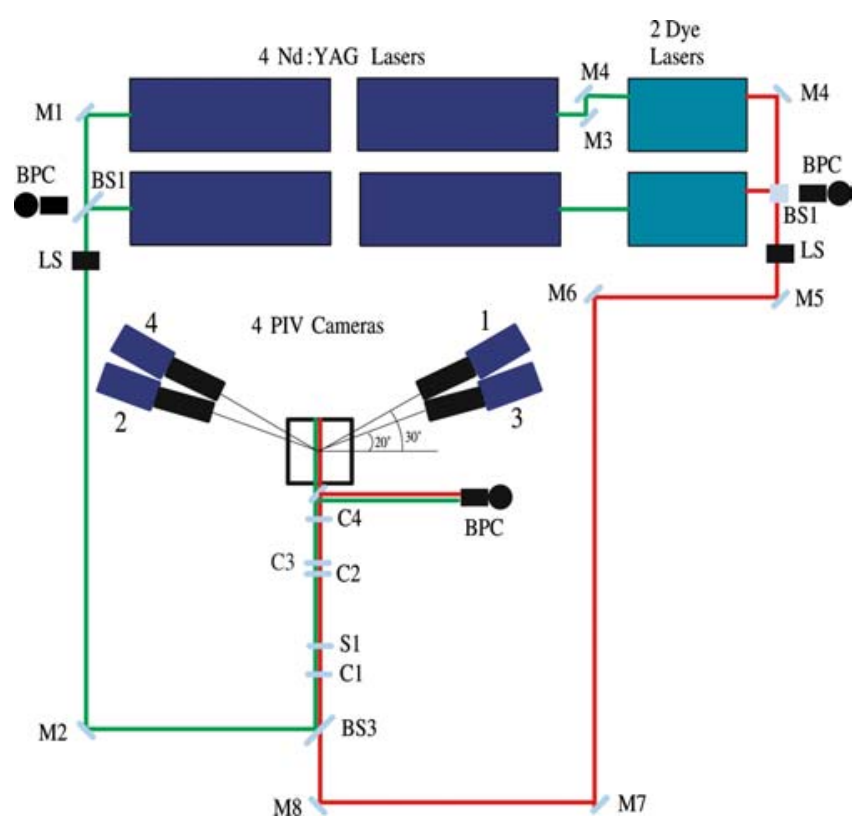

Fig. 2. Basic layout of the present frequency-based PIV system. Two Nd:YAG lasers provide the double-pulsed green light sheet and two additional Nd:YAG lasers pump two dye lasers to provide the double-pulsed red light sheet. Four PIV cameras are arranged in an asymmetric forward-forward scatter configuration

particles. Each individual particle image is a single-color, double-frame, single-exposure PIV image acquired at an angle to the light-sheet normal, thus, allowing two cameras oriented in a stereo configuration to determine the two inplane velocity components and the one out-of-plane velocity component over the measurement field-of-view. Each of the two individual stereo systems produces a single-color cross-correlation PIV measurement, and the two measurements are then mapped onto a common fieldof-view. Aluminum oxide particles with a nominal $0.5-\mu \mathrm{m}$ diameter are used here, for which the ratio of particle relaxation time and characteristic flow time gives a particle Stokes number that readily meets the requirements for assuring that the particles accurately follow the fluid motion (Samimy and Lele 1991; Clemens and Mungal 1991).

\section{1}

\section{DSPIV light sheet generation}

Two pairs of light sheets are created using four frequencydoubled Nd:YAG lasers. Two of these are sequentially triggered to create the double pulses for the $532-\mathrm{nm}$ sheets at $40 \mathrm{~mJ}$ per sheet. The other two are sequentially triggered at the same two instants of time to first produce 532$\mathrm{nm}$ pulses at $400 \mathrm{~mJ}$ per pulse, which then pump two pulsed dye lasers to provide the double pulses for the 635$\mathrm{nm}$ sheets at $40 \mathrm{~mJ}$ per sheet. The flashlamps and Qswitches are triggered by TTL signals at $10 \mathrm{~Hz}$, with a pulse duration of $10 \mathrm{~ns}$. The time delay $\Delta t$ between pulses is controlled by a PC-based programmable timing unit (PTU), controlled by the LaVision Davis 6.2 PIV acquisition software. All four lasers have a $100-n$ s intrinsic delay between the rising edge of the trigger pulse and the actual peak of the laser energy; this additional delay is negligible for the flow conditions used in the present study. The present $\Delta t \leq 95-\mu$ s pulse separations are sufficient to freeze the flow in the present turbulence measurements.

The 532-nm and 635-nm pulse pairs travel along independent optical paths until they are combined using a long-pass beamsplitter reflecting $>95 \%$ of the $532-\mathrm{nm}$ pulse and transmitting $>85 \%$ of the $635-\mathrm{nm}$ pulse. The pulse pairs then travel along a common sheet-forming optical path shown in Fig. 3 to create the differentially spaced sheets. The first two lenses, a concave-cylindrical $(f=100 \mathrm{~mm})$ and a convex-symmetric $(f=250 \mathrm{~mm})$, transform each round laser beam into a sheet with a width equal to the incident laser beam diameter and a height of $20 \mathrm{~mm}$. The next two lenses reduce the width of the nominal laser sheet by a factor of 10 , from $10 \mathrm{~mm}$ to $1 \mathrm{~mm}$, using a convex-symmetric $(f=250 \mathrm{~mm})$ and a plano-convex $(f=25 \mathrm{~mm})$ lens pair that form a reversed (10:1) Galilean telescope. The third set of optics consists of two plano-convex $(f=60 \mathrm{~mm}$ and $f=80 \mathrm{~mm})$ lenses separated by $160 \mathrm{~mm}$ to produce an effective focal length of $f=370 \mathrm{~mm}$, which creates a waist in the test section.

The optics are arranged on a single optical rail to provide a common optical axis, with each optic mounted on a micrometer stage to allow individual adjustments along and transverse to the optical axis. This allows the laser sheet waist to be moved within the test section. Due to the different frequencies of the light sheets, their respective waists fall at slightly different locations. To match the waists of the $532-\mathrm{nm}$ and $635-\mathrm{nm}$ sheets, the diameters of the $635-\mathrm{nm}$ laser beams were increased to create the correct input diameter into the sheet forming optics, repositioning the $635-\mathrm{nm}$ waist location to that of the 532-nm location.

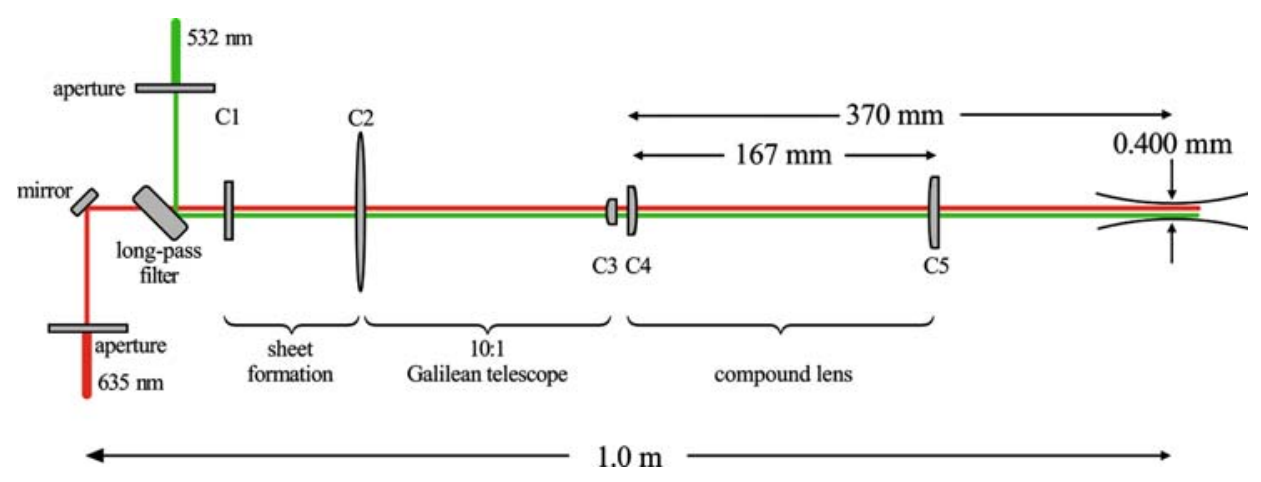

Fig. 3. Optical components used to combine the green and red laser pulses to create the two differentially spaced double-pulsed light sheets in Fig. 1 

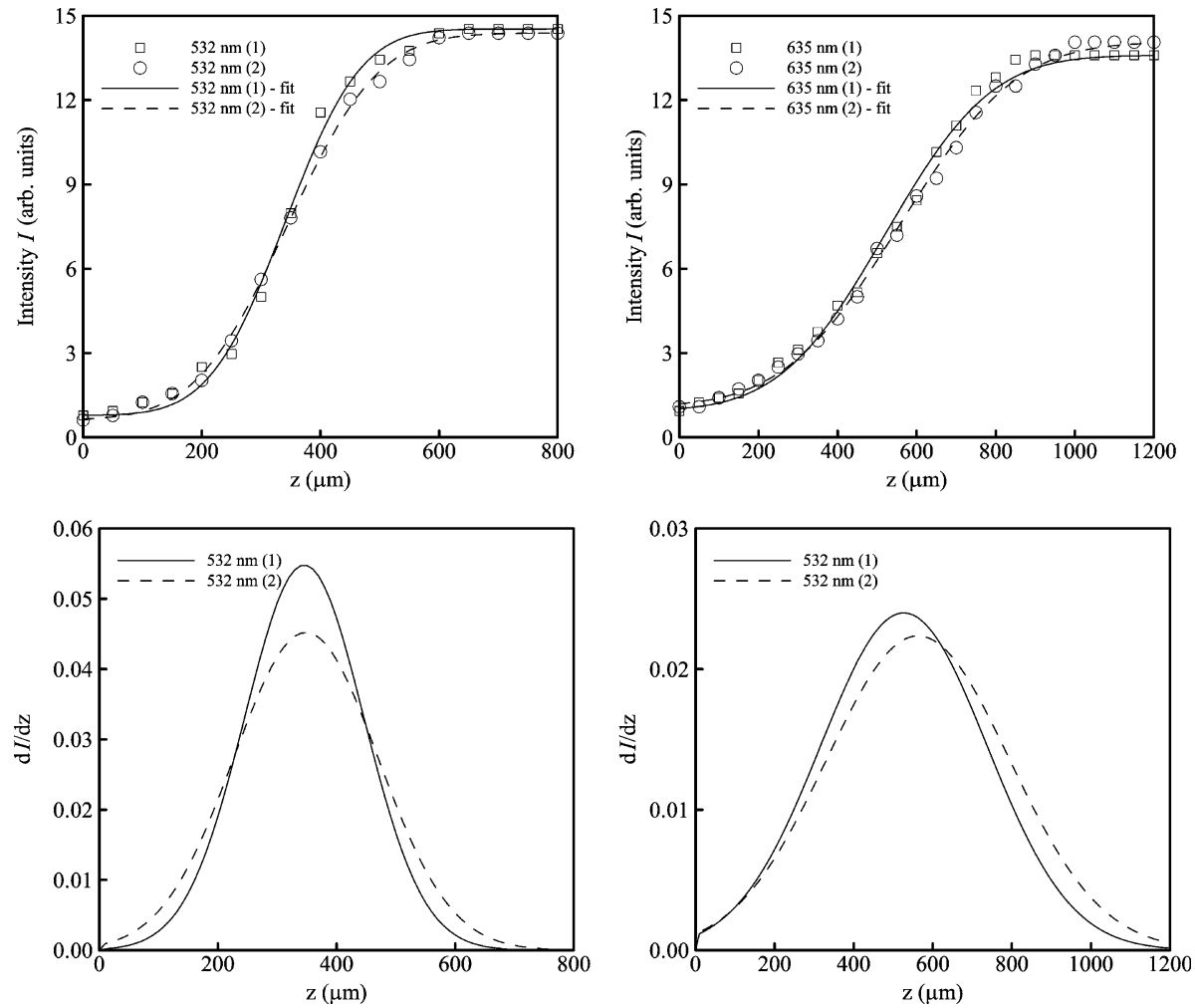

Fig. 4. Typical results for laser sheet profile measurements for the two green sheets (left) and the two red sheets (right), showing raw measured values (symbols) and error function fits (lines) at the top, and corresponding derivatives giving sheet intensity profiles at the bottom

\section{2}

\section{Light sheet characterization and alignment}

The thickness, spacing, and two-axis parallelism of the two pairs of differentially spaced light sheets contribute directly to the accuracy achievable by DSPIV measurements. The thicknesses in part determine the spatial resolution of the measurements. In the present system, these thicknesses were measured in the center of the field-of-view by traversing a knife edge across each sheet and collecting the transmitted light onto a photodiode detector. To quantify each sheet thickness, as shown in Fig. 4, an error function was fitted using a nonlinear least-squares match to the measured profile, and then differentiated to obtain a sheetnormal Gaussian intensity profile. The three lowest-order moments computed from this Gaussian profile allow the centerline position, the local intensity maximum, and the local $1 / \mathrm{e}^{2}$ thickness of each laser sheet to be determined. Typical results are shown in Fig. 4. It is apparent that the measured intensity profiles across the $532-\mathrm{nm}$ and $635-\mathrm{nm}$ light sheets are almost Gaussian, and, the resulting $1 / \mathrm{e}^{2}$ values thus provide a reasonable characterization of the light sheet thicknesses. The above procedure was also repeated at the right and left edges of the field-of-view to verify that the thickness of the four respective light sheets does not vary significantly over the entire field-of-view of the measurements.

The coincidence of each of the 532-nm and 635-nm light sheet pairs is essential to minimize the loss of particle pairs due to sheet misalignment, while the separation between the 532-nm and 635-nm light sheets determines the $\Delta z$ value involved in $z$ derivative components of the velocity gradient tensor $\partial u_{i} / \partial x_{j}$. Errors in the separation distance, or variations in this separation over the fieldof-view due to nonparallelism of the sheets, will directly affect the accuracy of the derivatives in the out-of-plane direction. An experimental method was devised to quantitatively measure the respective laser sheet coincidence, separation distance, and sheet non-parallelism. This procedure for aligning the respective laser sheet pairs initially aligns the $532-\mathrm{nm}$ and $635-\mathrm{nm}$ beam pairs to be coincident over the length of the optical path. The coincidence is checked optically using CCD cameras, denoted BPCs (beam pair coincidence) in Fig. 2, and a centroid-finding image processing routine based on the beam spot intensities that can achieve alignment of the centroids to within $\pm 25 \mu \mathrm{m}$. The long optical path ensures that the beams, once aligned, remain parallel throughout the test section. The respective optical paths are aligned individually, without the sheet-forming optics, allowing a minimum number of optical adjustments to overlap the centroids of the laser beams. The beams are then formed into light sheets by inserting the spherical and cylindrical lenses.

The procedure for separating the two measurement planes begins with the two light sheet pairs initially coincident. The $635-\mathrm{nm}$ sheets are then moved by rotation of a mirror in the $z$ direction to create the necessary separation. A small fraction $(<5 \%)$ of the light sheet energy in each of the two 532-nm and the two 635-nm sheets is picked off prior to the test section and used with a semitransparent target to create an image of the sheet crosssection on the BPC camera. The imaging target is located at the same distance from the pick-off as the measurement location in the test section. From these images, intensity profiles are obtained by averaging every 16 rows, and a Gaussian fit is made using the same procedure noted above. From this Gaussian fit, the center location of the intensity profile is obtained for each of the four laser sheets. Figure 5 shows how the respective laser sheet 
centroids vary along the vertical direction, and verifies that each sheet pair is essentially coincident, and that the spacing between the 532-nm and $635-\mathrm{nm}$ planes is essentially uniform along the vertical direction.

The four light sheets must also remain parallel over the entire field-of-view of the measurement. This parallelism is assessed as shown in Fig. 5 by conducting the procedure described above at the left $(y=-10 \mathrm{~mm})$ and right $(y=$ $+10 \mathrm{~mm}$ ) edges of the field-of-view, in addition to the center $(y=0 \mathrm{~mm})$ of the field-of-view. The results verify that the $532-\mathrm{nm}$ and $635-\mathrm{nm}$ sheets are parallel to within slightly less than $1^{\circ}$.

\section{3}

\section{DSPIV imaging arrangement}

Scattered light from the seed particles is recorded on four interline transfer CCD cameras with a 200 -ns interframe timing. The $1280 \times 1024$-pixel arrays provide sufficient spatial dynamic range in the particle images, and the 12bit signal depth provides sufficient signal dynamic range. The present field-of-view is $15.5 \times 12.5 \mathrm{~mm}$, giving a magnification of 0.55 based on the physical size of the CCD chip $(8.6 \times 6.8 \mathrm{~mm})$. Each camera uses a Sigma 70-300 f/45.6 APO macro lens to allow up to 1:1 imaging at a minimum focal length of $40.1 \mathrm{~cm}$ to achieve the desired fieldof-view. The 532-nm camera pair has narrow-band filters centered at $532 \pm 5 \mathrm{~nm}$ that block the 635 -nm light, and the 635-nm cameras are equipped with OG570 Schott glass filters that effectively block the 532-nm light, isolating the scattered light from the laser sheets onto the appropriate camera pairs. The use of interline transfer CCD cameras allows for single-color, cross-correlation PIV analysis of the resulting particle images. The present DSPIV system uses four PCO SensiCam cameras operating simultaneously in double-frame mode, so the net throughput to the hard drive is approximately 1 frame/s.

The four cameras are arranged in an asymmetric angular-displacement configuration, shown in Fig. 6, with each of the camera pairs satisfying the Scheimpflug condition for stereoscopic imaging (Prasad and Jensen 1995). The small field-of-view of the measurements, coupled with the long focal length of the camera lens, dictates that a large $f \#$ aperture must be used to create particle images that are sufficiently focused across the full extent of the field-of-view. To obtain precise alignment between the object, lens, and image planes while maintaining a coincident field-of-view for all cameras, specially designed camera mounts are attached to rails providing forwardbackward and side-to-side adjustments, along with fine vertical adjustment and a smooth angular adjustment.

In the present camera configuration, each pair of stereo cameras is orientated as shown in Fig. 6 so that the included angle between the optical axes of each camera pair is $50^{\circ}$. A more traditional symmetric forward-backward camera configuration, in which one camera in each pair images the particles in backward scattering mode while the second camera images in forward scattering mode, was not implemented here due to the decreased signal-to-noise level that results in backward scattering and the limited energy available in the laser sheets. In the forwardforward camera configuration used here, one camera from each pair was arranged with a viewing angle of $20^{\circ}$ and the other with a viewing angle of $30^{\circ}$.

System performance studies of such angulardisplacement stereo PIV systems by Lawson and $\mathrm{Wu}$ (1997a, 1997b) were used as a guide in determining the present camera system configuration. The error ratio, defined as the ratio of the out-of-plane rms displacement

$$
\begin{array}{ll}
\text { 口 } 532 \mathrm{~nm}(1) & \diamond 635 \mathrm{~nm}(1) \\
\circ 532 \mathrm{~nm}(2) & \triangleright 635 \mathrm{~nm}(2)
\end{array}
$$
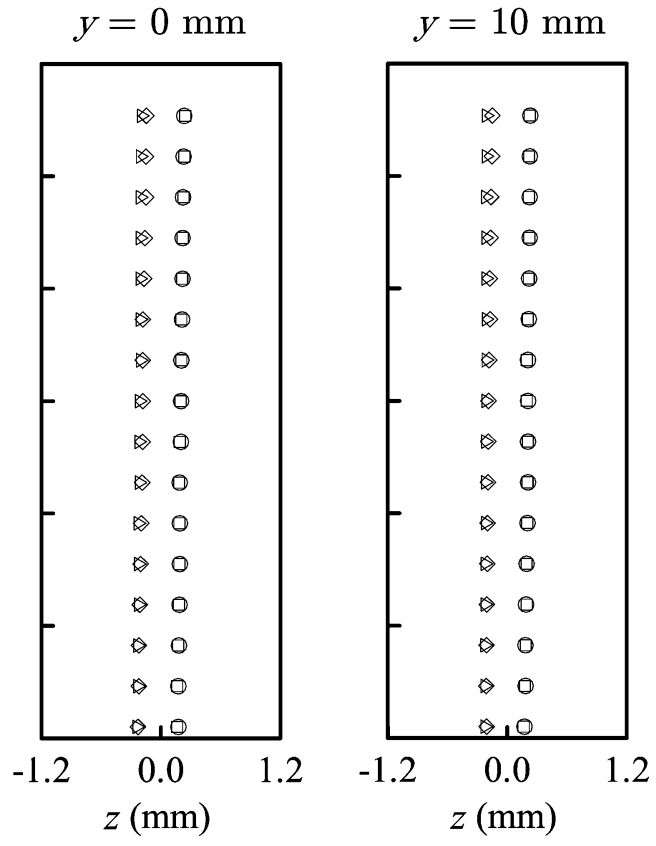

Fig. 5. Centroid positions of the four laser sheets measured at the left edge (left), at the center (center), and at the right edge (right) of the field-of-view, verifying laser sheet coincidence, separation, and parallelism 


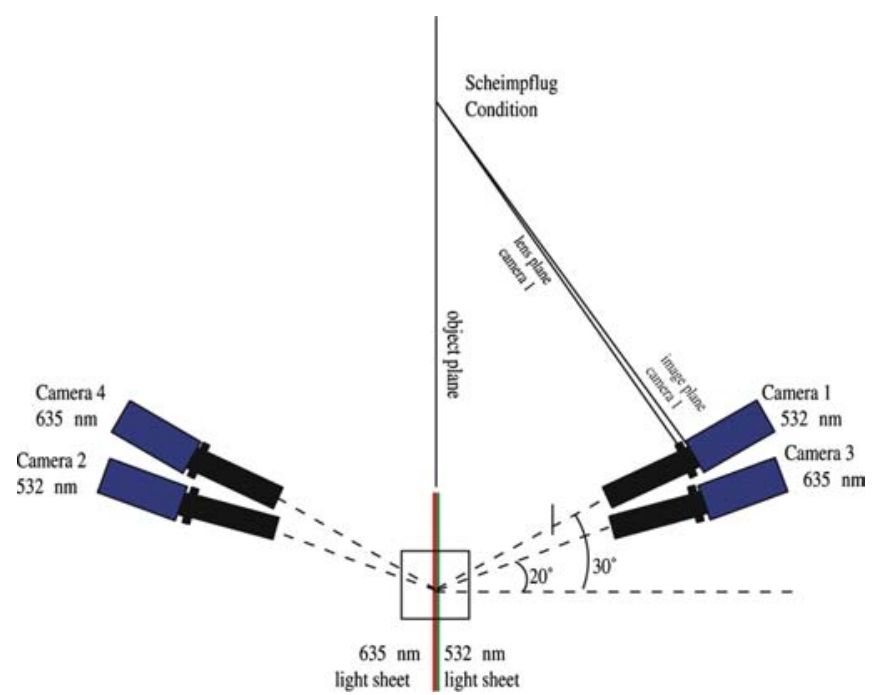

Fig. 6. Asymmetric forward-forward scatter configuration of the four PIV cameras. Each camera pair has one camera at $30^{\circ}$ and one camera at $20^{\circ}$ relative to the light sheet normals

error to the in-plane rms displacement error, is used to determine the optimal camera configuration. The optimal error ratios occur for included angles between $40^{\circ}$ and $50^{\circ}$. For the present DSPIV configuration, an error ratio of 1 to 3 is expected, based on the included angle of $50^{\circ}$. The results of Coudert et al. (2000) for asymmetric camera configurations indicate that, for the present $50^{\circ}$ included angle and $10^{\circ}$ asymmetry angle, the error ratio is expected to be about 1 to 2 . This error ratio is similar to the values obtained by Lawson and Wu (1997b) for a symmetric arrangement, indicating that effects of the asymmetry in the camera arrangement are essentially negligible for the present small asymmetry angle.

\section{4}

\section{DSPIV particle image processing}

The particle images were processed on a dual-board PC with a $500-\mathrm{mHz}$ CPU and $1 \mathrm{~GB}$ RAM using the LaVision Davis 6.2 software, based on a cross-correlation method with a fast Fourier transform (FFT) based algorithm (Raffel et al. 1998). The software employs an adaptive multipass technique to calculate the velocity field, using $32 \times 32$-pixel interrogation boxes on the first pass and then refining the correlation peak search on a second $32 \times 32$ pixel interrogation pass. This technique produces a final in-plane spatial resolution of $400 \mu \mathrm{m}$, based on the physical pixel size of the CCD array, the magnification, and the interrogation box size. No overlap is used in the final vector field, allowing the in-plane spatial resolution to match the out-of-plane spatial resolution between the planes. The velocity fields were then further processed to replace spurious vectors (typically less than $10 \%$ of total vectors) by interpolation with a $3 \times 3$ median filter, and were subsequently processed with a $3 \times 3$ median smoothing filter to reduce high-frequency noise.

The velocity gradients were computed using linear central-differencing in the $x$ and $y$ directions and onesided differencing in the $z$ direction of the measured velocity fields to obtain all nine components of $\partial u_{i} / \partial x_{j}$. The gradient components were calculated on a standard $3 \times 3$ template that incorporates two independent coordinate frames, oriented at $45^{\circ}$ relative to each other, to provide two separate estimates of the gradient components, which were then averaged to obtain the final values. This procedure uses all eight surrounding data points for the inplane gradient components, and all five surrounding data points for the out-of-plane gradient components, to reduce random error in the resulting gradient components without the resolution degradation that would result from a simple explicit filter that gives the same noise reduction.

\section{5 \\ DSPIV measurement resolution}

Four principal factors contribute to the spatial resolution of these DSPIV measurements: (1) the laser sheet thickness and separation; (2) the PIV correlation window size; (3) the typical bad-vector replacement region size; and (4) the PIV smoothing filter scale. The laser sheet thicknesses were measured, as discussed in Section 2 , as $\lambda_{1} \approx 400 \mu \mathrm{m}$ for the $532-\mathrm{nm}$ sheets and $\lambda_{1} \approx 800 \mu \mathrm{m}$ for the $635-\mathrm{nm}$ sheets. The 32×32-pixel PIV correlation window, together with the pixel size and magnification ratio, effectively filters the velocity field at a scale $\lambda_{2} \approx 400 \mu \mathrm{m}$. Based on the spacing of the vectors, the bad-vector replacement scale is $\lambda_{3} \approx 800 \mu \mathrm{m}$, and the explicit filtering in the final processing stage is at a scale $\lambda_{4} \approx 800 \mu \mathrm{m}$. Since these contributions to the net resolution are independent, the resulting velocity gradient fields are resolved to a length scale of $\lambda_{\text {net }} \equiv\left(\lambda_{1}{ }^{2}+\lambda_{2}{ }^{2}+\lambda_{3}{ }^{2}+\lambda_{4}{ }^{2}\right)^{1 / 2} \approx 1,400 \mu \mathrm{m}$.

\section{3}

\section{DSPIV system calibration}

The accuracy of any stereo PIV measurement, as well as the present DSPIV measurements, depends crucially on the quality of the particle images, the accuracy of the inferred particle displacements, and the extent to which the particles follow the fluid motion. The two former issues depend principally on focusing aberrations and distortions inherent in the imaging of the particles. Focusing aberrations mainly influences the particle image size, with a general rule (Adrian 1997; Raffel et al. 1998) being that the particle images should typically span 2-3 pixels on the CCD array. For smaller particle images, it becomes difficult to obtain a sub-pixel estimate of the particle centroid location, and as the particle images become much larger, it eventually becomes difficult to accurately determine the centroid displacements. For the present DSPIV system, the individual particle images typically span $4-5$ pixels, which provides sufficient accuracy in determining the centroid displacements.

Stereoscopic imaging requires a calibration that incorporates the registration of the camera pairs and their mapping from the object plane onto the image plane to correct for distortions due to variable magnification across the image. For the present DSPIV system, each independent camera pair must be registered and mapped as usual for any stereo PIV system, followed by the additional step of registering and mapping both camera pairs into a single reference frame. By far the largest source of image distortion inherent in stereoscopic imaging results from the 
variable magnification $M$ across the image plane introduced by the off-axis viewing angle of the cameras. A simple relation between particle displacements $\left(X_{1}, X_{2}\right)$ in the object plane and their displacements $\left(x_{1}, x_{2}\right)$ in the image plane of the form $\left(X_{1}, X_{2}\right)=-M\left(x_{1}, x_{2}\right)$, where $M$ is the magnification, is no longer valid and instead takes the form $X=1 \mathscr{F}(x)$, where $1 \mathscr{\mathscr { F }}$ is a nonlinear mapping function created from images of a calibration target. This mapping function accounts for the distortion and provides the third velocity component from the apparent in-plane components.

The stereo calibration method of Soloff et al. (1997) was implemented to calculate these mapping functions for the four cameras. This procedure eliminates the need to physically register each camera, allowing for a far simpler mathematical registration. This is important for DSPIV measurements, since four cameras must be precisely aligned to the same physical field-of-view. The procedure used a precision calibration target with micrometer-controlled translation stages. A reference point on the target defines the origin for all the calibration images. From the four calibration images of this target, the camera magnification factors and their positions are compared, and the necessary adjustments are made to allow precise overlap of their respective fields-of-view. Since this requires good focus across the target, large lens aperture settings are used along with the Scheimpflug condition.

The precision calibration target was formed from a regular grid of white crosses on a black background. The camera configuration requires that the target must be transparent, and the field-of-view dictates a target size of approximately $20 \times 20 \mathrm{~mm}$. The number of crosses directly affects the accuracy of the mapping-function polynomial. With too few crosses, a coarse fit is obtained, while too many crosses makes it difficult to obtain a smooth fit. The spacing between crosses must be precisely known, and the cross thickness and length determine the accuracy of their centroid identification. To create this target, a $225 \times 280$ $\mathrm{mm}$ calibration target was first printed and then photographed onto a $35-\mathrm{mm}$ film negative with a known magnification. The resulting $35-\mathrm{mm}$ negative provided a very accurate calibration target, which was verified under a microscope to give a cross spacing of $660 \mu \mathrm{m}$.

The elegance of the Soloff et al. (1997) calibration method is that only three numbers are required as inputs to compute the registration and mapping functions for each of the four cameras. These numbers are the distance between the crosses on the calibration target, the distance between the first and second calibration planes, and the distance between the differentially spaced light sheets. From this information, the camera positions, angles, and magnifications are calculated, along with the mapping functions. The camera positions and angles were iteratively changed based on the computed magnification, position, and angle until optimum calibration and focus were obtained.

The procedure described above involves two independent stereoscopic calibrations. In a DSPIV system, a further mapping is required to relate vectors from one stereo system to the other. The relative positions of the calibration target used for this DSPIV calibration are illustrated in Fig. 7. The target locations are near the front and back planes of each of the laser sheets. The target, thus, has to be displaced only twice, with the middle target location being used for both the 532-nm and the 635-nm sheet calibrations. By restricting the procedure to two target displacement steps, and by using the micrometer-controlled calibration apparatus, a net displacement accuracy of $1.5 \mu \mathrm{m}$ was obtained.

The calibration was carried out by taking the first two images for the 532-nm cameras and then displacing the calibration target by a known distance $\Delta z$ with a micrometer translation stage. The second two images were then obtained with the 635-nm cameras and the mapping functions generated. With the eight target images, two for each of the four cameras, the DSPIV mapping functions for each camera were created by first locating the positions of the crosses with respect to the image origin using a centroid identification algorithm. Once these grid locations were defined, a two-dimensional third-order polynomial was generated at each point using least-squares error minimization to create the in-plane mapping function for each camera. To construct the mapping for the out-of-plane component, the difference in the mappings between the two calibration planes for each camera pair is used.

\section{4}

\section{DSPIV calibration and measurement errors}

This section examines errors associated with the DSPIV calibration procedure described above, and presents several experimental methods for determining the resulting accuracy of velocity differences obtained from the measurements themselves.

\section{1 \\ Calibration errors}

Since the calibration procedure generates the mapping functions that relate particle displacements to velocity vectors, it is essential to understand the error sources that can arise in this calibration process. These consist of: (1) errors in the cross spacing on the calibration target; (2) errors introduced by approximating the true mapping function with a third-order polynomial; (3) errors introduced by imperfect parallelism of the calibration planes relative to each other; (4) errors due to the calibration planes not being precisely parallel to light sheets; and (5) errors introduced by uncertainties in the absolute positions of the calibration planes within each light sheet.

Since both the cross separation on the original largescale printed target and the magnification factor used to

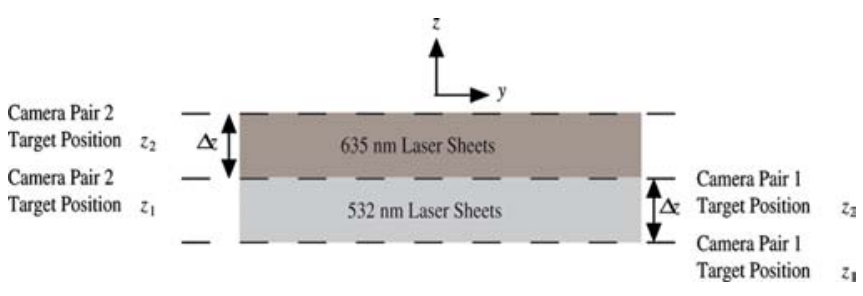

Fig. 7. Relative positions of calibration target at front and back of green $(532-\mathrm{nm})$ and red $(635-\mathrm{nm})$ light sheets 

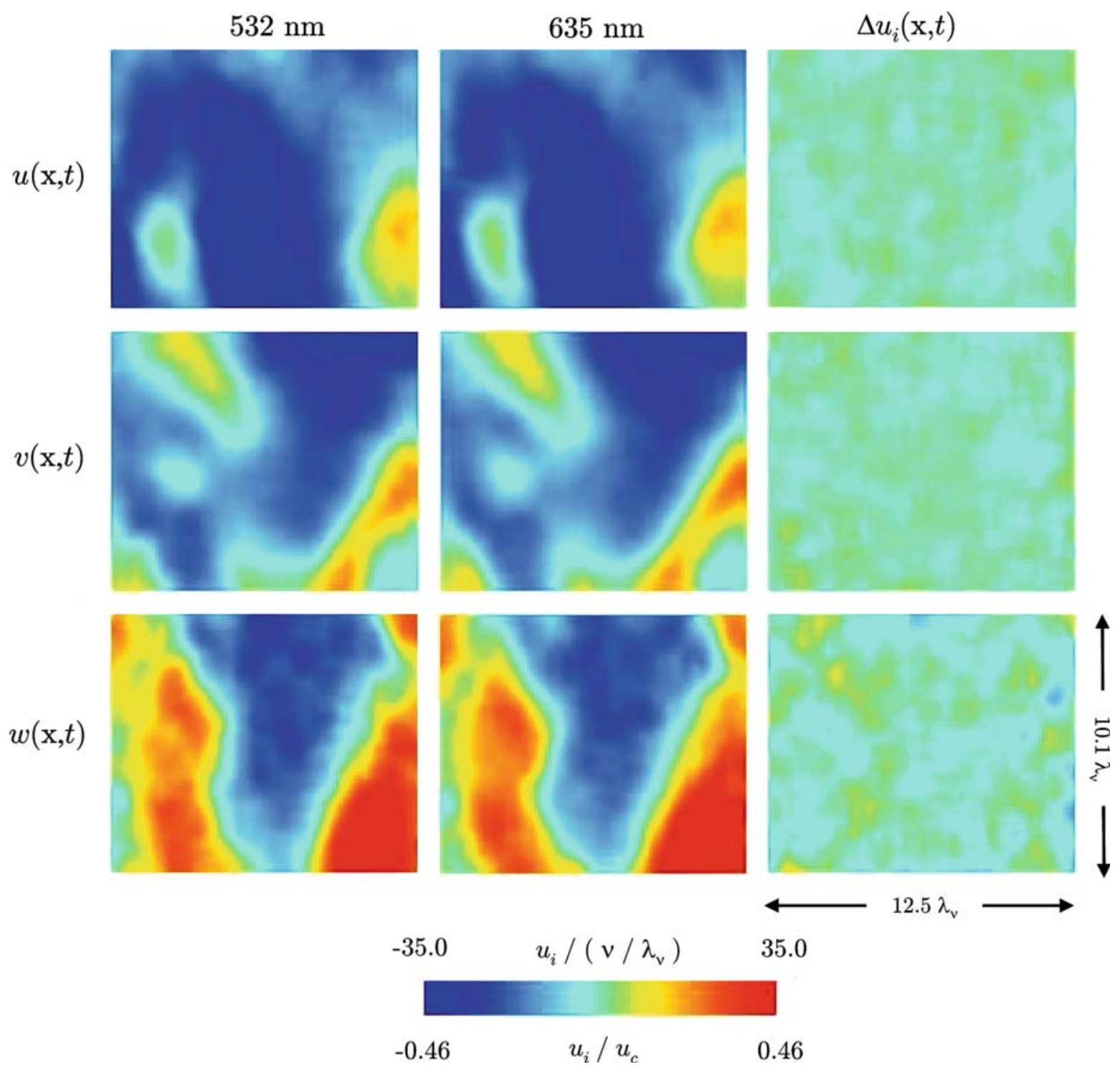

Fig. 8. Typical instantaneous velocity component fields $u_{i}(\mathbf{x}, t)$ from coincident-plane imaging tests, showing independently measured fields from 532-nm (left) and 635-nm (middle) stereo camera pairs, and corresponding difference fields $\Delta u_{i}(\mathbf{x}, t)($ right $)$

reduce this to the $35-\mathrm{mm}$ film negative are accurately known, the resulting uncertainty in the cross spacing on the target is only about $10 \mu \mathrm{m}$. Moreover, the relatively large number of crosses on the target $(\geq 250)$ leads to a net calibration error that becomes negligible in comparison with other error sources. Errors due to the mapping function being locally approximated by a two-dimensional third-order polynomial are known to be negligible for imaging configurations where severe distortions are not present (Soloff et al. 1997). For the present asymmetric forward-forward imaging arrangement with camera angles of $20^{\circ}$ and $30^{\circ}$, the resulting errors from the polynomialbased mapping function are negligible. The average deviation between the measured and calculated positions of the crosses on the calibration target is found to be 0.3 pixels; this is significantly better than the 0.4-0.6-pixel criterion often used to characterize a good calibration. Soloff et al. (1997) have further shown that the rms error from the polynomial mapping function should be no larger than the error in locating the particle centroids, which Prasad et al. (1992) have shown to be $5-10 \%$ of the particle diameter.

For the present target and calibration procedure, the resulting measured rms error from the polynomial mapping function corresponds to just $3.6 \%$ of the particle diameter, is substantially smaller than the error associated with determining the particle centroids.

Moreover, since the calibration procedure uses micrometer stages having $1.5-\mu \mathrm{m}$ accuracy, errors introduced by any nonparallelism between the calibration target planes should be of comparable order also negligible. The $1.5-\mu \mathrm{m}$ accuracy of the micrometer stages gives an error of, at most, $0.3 \%$ in the spacing between the parallel calibration planes based on the $400-\mu \mathrm{m}$ inter-plane separation. The largest error source comes from the requirement that the calibration target should be precisely parallel to the light sheets. If the calibration target plane is instead at some 


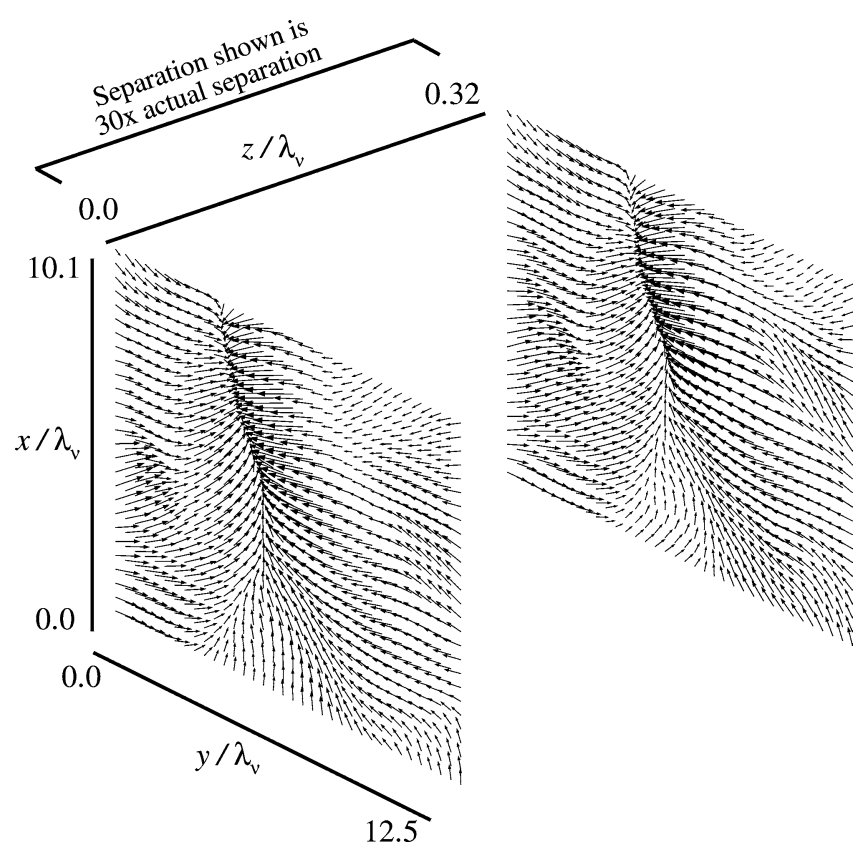

Fig. 9. Typical instantaneous velocity vector fields $\mathbf{u}(\mathbf{x}, t)$ from DSPIV measurements showing velocity fields in two planes with $400-\mu \mathrm{m} z$ separation. Interplane separation is shown greatly exaggerated; the actual separation between planes is the same as the vector-to-vector spacing within each plane

small angle with respect to the light sheet plane, then the mapping functions are appropriate for the velocity field in the rotated coordinate system and not the coordinate system of the light sheet. Until very recently, there has not been a method to calculate this error; Bjorkquist (2002) and Wieneke (2003) suggest a numerical re-calibration to quantify and cancel out this effect in a post-processing manner, but this procedure has not been used here.

\section{2}

\section{Measurement errors}

A coincident light-sheet method was devised to allow assessment of the final DSPIV measurement accuracy. This quantitative assessment of accuracy uses both stereo camera pairs arranged to image the same particle field by aligning the $532-\mathrm{nm}$ and $635-\mathrm{nm}$ light sheets to be as nearly coincident as possible with the present optical system. Differences in the two resulting independent velocity fields $\mathbf{u}(\mathbf{x}, t)$ from such coincident-plane imaging tests then reflect fundamental limits on the accuracy with which velocity gradients can be measured by the DSPIV system. The two leftmost columns in Fig. 8 show a typical example of the three velocity component fields $u_{i}(\mathbf{x}, t)$ obtained independently from the $532-\mathrm{nm}$ and $635-\mathrm{nm}$ camera pairs in such coincident-plane tests. In principle, the component fields should be identical, with any differences resulting from the cumulative effects of errors considered above and the additional errors introduced by the independent light sheet generation and positioning. The right column shows the resulting differences $\Delta u_{i}(\mathbf{x}, t)$ between the respective component fields on the same quantitative color scale. It is evident that the $w$ component differences are typically larger than the $u$ and $v$ component differences, due to the inherently larger errors in mea- suring the out-of-plane velocity with stereo PIV systems. However, it is apparent for all three velocity components that the differences $\Delta u_{i}$ are far smaller than the measured component values themselves, quantifying the high accuracy of these velocity field measurements.

5

\section{DSPIV measurement demonstration}

In contrast to the coincident-plane validation measurements above, in this section, the 532-nm and $635-\mathrm{nm}$ light-sheet planes are arranged with a differential spacing $\Delta z$ $>0$ and are used to demonstrate DSPIV measurements of velocity gradients at the intermediate and small scales of a turbulent jet flow with local outer-scale Reynolds number $R e_{\delta}=6,000$. To resolve essentially all scales of the fluid motion, $\Delta z$ must be set comparable to the local inner (viscous) length scale $\lambda_{v}$ of the turbulent flow. As $\Delta z$ is made successively smaller, the velocity fields in the two planes become nearly identical and the individual component differences eventually become dominated by the intrinsic error limits in Fig. 8. Here, the two planes are separated in the $z$ direction by $400 \mu \mathrm{m}$, with the resulting $\left(\Delta z / \lambda_{v}\right)=0.32$.

Figure 9 shows a typical example of the instantaneous velocity vector fields $\mathbf{u}(\mathbf{x}, t)$ measured in the two differentially spaced planes, with the dimensions shown normalized by the local inner length scale $\lambda_{v}$. The separation between the planes is shown greatly exaggerated to allow comparisons of the vector fields in both planes; the actual separation between the two planes is the same as the vector-to-vector spacing within each plane. The relatively small differences in the vectors between the two planes are due to this differential $z$ spacing. It is these differences that give rise to the $z$ derivative components of the resulting velocity gradient tensor fields $\partial u_{i} / \partial x_{j}$.

Figure 10 further shows a typical example of all three simultaneously measured instantaneous velocity component fields $u_{i}(\mathbf{x}, t)$ in the two differentially spaced $532-\mathrm{nm}$ and $635-\mathrm{nm}$ light-sheet planes. The mean value of each velocity component has been subtracted to allow direct comparisons between the spatial structure in the $u, v$, and $w$ component fields. The dimensions of each plane are indicated in terms of the local inner length scale $\lambda_{v}$, and the color bar gives the velocity component values normalized by the local outer (centerline) velocity scale $u_{\mathrm{c}}$, as well as by the local inner velocity scale $\left(v / \lambda_{v}\right)$. Small differences discernible between the independent $u_{i}(\mathbf{x}, t)$ fields in the 532-nm and 635-nm planes, due to the differential $z$ spacing, produce the $z$ derivative components in the resulting velocity gradients $\partial u_{i} / \partial x_{j}$.

Figure 11 shows a typical example of the measured instantaneous velocity gradient tensor fields $\partial u_{i} / \partial x_{j}$ from such frequency-based DSPIV measurements, corresponding to the velocity component fields shown in Fig. 10. The color bar gives the velocity gradient component values normalized by the local outer velocity gradient scale $\left(u_{\mathrm{c}} / \delta\right)$ and by the local inner velocity gradient scale $v / \lambda_{\mathrm{v}}{ }^{2}$. In principle, the inner-variable scalings should become $R e_{\delta}$-independent at sufficiently large values of $R e_{\delta}$, while the outer-variable scalings will show the increasing intermittency with increasing $R e_{\delta}$. 

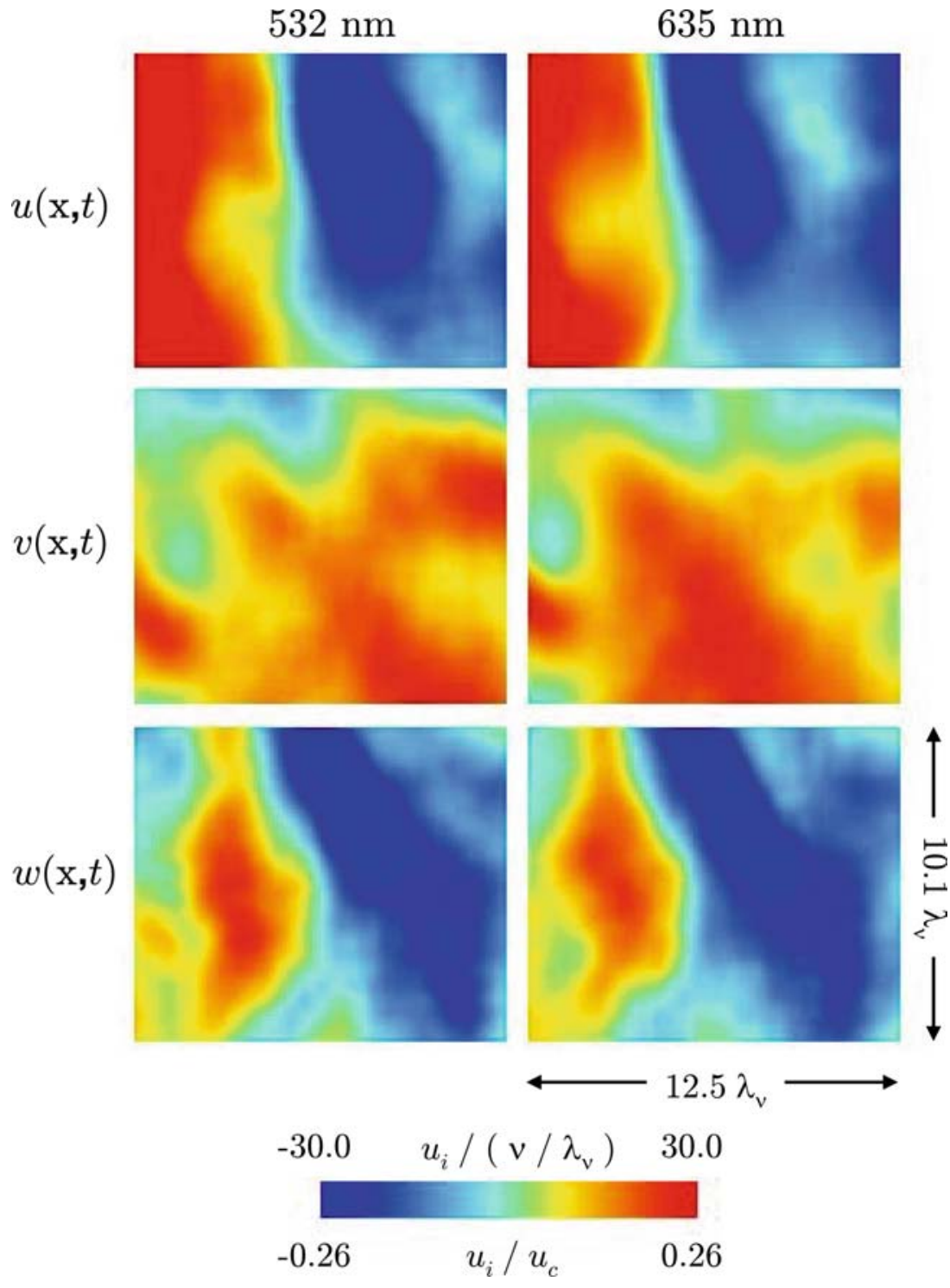

Fig. 10. A typical example of an instantaneous velocity component field $u_{i}(\mathbf{x}, t)$ showing velocity fields in the 532-nm (left) and 635-nm (right) planes separated by $\Delta z=0.32 \lambda_{v}$. The field-of-view is shown normalized by the local inner length scale $\lambda_{v}$. The color scale shows values normalized on the local inner $\left(v, \lambda_{v}\right)$ and outer $\left(u_{c}, \delta\right)$ scales
The nine simultaneously measured velocity gradient components $\partial u_{i} / \partial x_{j}$ in turn allow various fields essential to modeling the intermediate and small scales of turbulence, including the vorticity field, the strain rate field, and the kinetic energy dissipation rate field, to be obtained.

These results demonstrate the successful realization and fundamental characterization of a new two-

frequency DSPIV technique that permits the first direct, noninvasive, simultaneous, highly resolved measurements of all nine components of the velocity gradient tensor field at the small scales of shear flow turbulence. The present results have focused on the description and characterization of the measurement technique itself. Mullin and Dahm (2004a, 2004b) present additional extensive results from the application of this technique to a turbulent shear flow in a highly detailed study into the effects of the local strain rate in shear flow turbulence on the structure, statistics, similarity, and scaling of velocity gradient fields at the inner scales of a turbulent flow.

\section{6}

\section{Conclusions}

Dual-plane stereo particle imaging velocimetry (DSPIV) using two different light-sheet frequencies allows direct, nontrusive measurements of all nine simultaneous components of the velocity gradient tensor fields $\partial u_{i} / \partial x_{j}$ at the intermediate and small scales of turbulent flows. The use of two different laser frequencies, in conjunction with filters to separate the scattered light from seed particles onto the individual stereo camera pairs, allows traditional solid metal oxide particles to be used as the seed, and, thereby, permits such DSPIV measurements to be made in exothermic reacting flows as well as in nonreacting flows. Unlike earlier dual-plane measurement approaches, the present two-axis asymmetric forward-forward imaging configuration allows the increased scattering intensities associated with forward imaging while minimizing the ratio of out-of-plane to in-plane velocity component errors. Moreover, by properly arranging the components of such a DSPIV system, it is possible to resolve the quasi-universal intermediate and small scales of all 

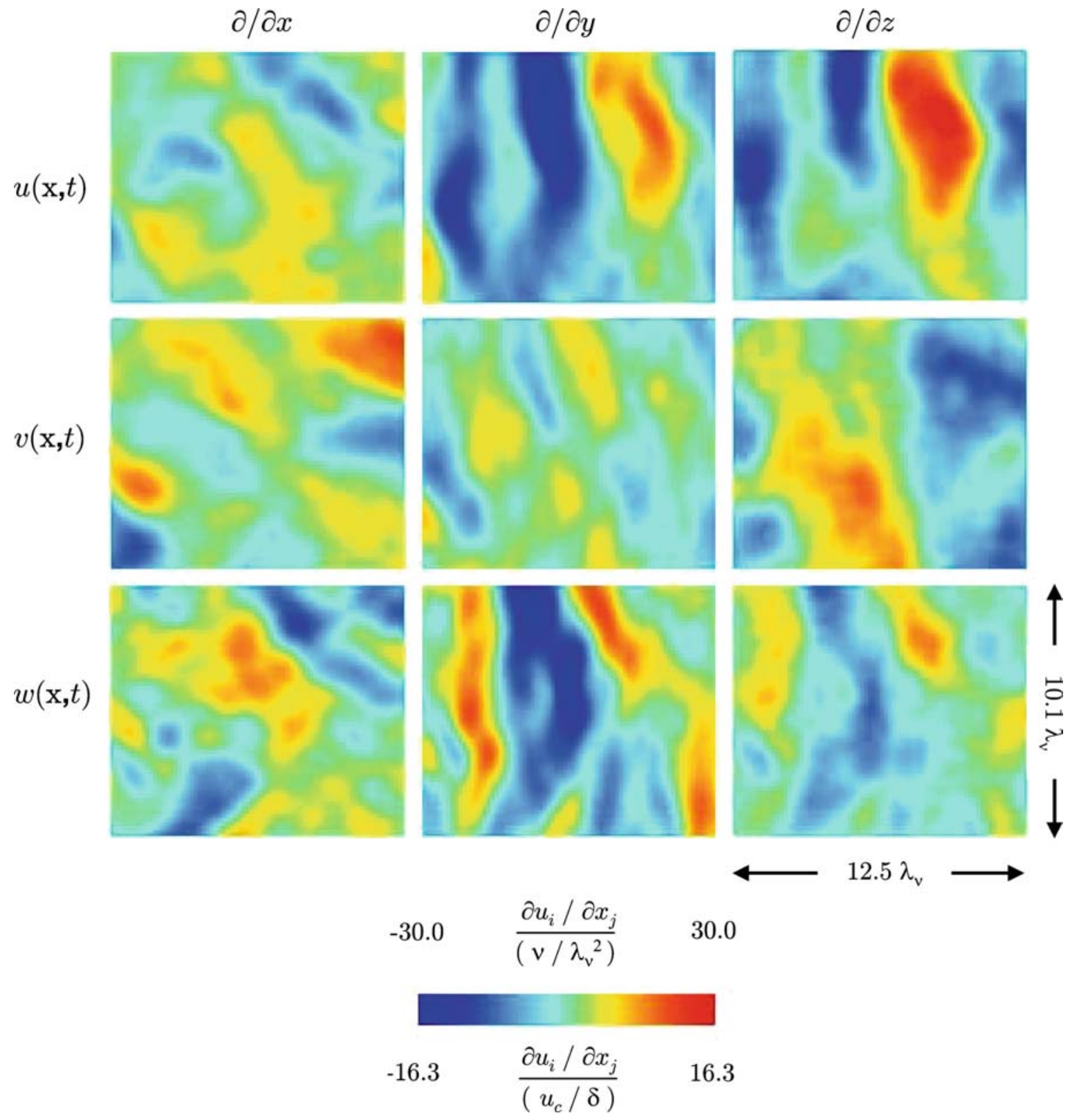

30.0

Fig. 11. Typical example of the full nine-component velocity gradient tensor field $\partial u_{i} / \partial x_{j}(\mathbf{x}, t)$. The field-of-view is shown normalized by local inner length scale $\lambda_{v}$. The color scale shows quantitative values normalized both on the local inner $\left(v, \lambda_{v}\right)$ and outer $\left(u_{\mathrm{c}}, \delta\right)$ scales

velocity gradient components in turbulent flows, allowing direct experimental study of gradient fields such as the vorticity vector field, the strain rate tensor field, and the true kinetic energy dissipation rate field in turbulent flows. The results presented here describe the key components of such a two-frequency DSPIV system and demonstrate the successful realization and characterization of such a system to obtain simultaneous highly resolved measurements of all nine components of the velocity gradient tensor field at the small scales of a turbulent shear flow.

\section{References}

Adrian RJ (1997) Dynamic ranges of velocity and spatial resolution of particle image velocimetry. Meas Sci Technol 8(12):1393-1398

Antonia RA, Shah DA, Brown LWB (1987) Spectra of velocity derivatives in a turbulent wake. Phys Fluids 30(11):3455-3462

Antonia RA, Zhou T, Zhu Y (1998) Three-component vorticity measurements in a turbulent grid flow. J Fluid Mech 374:29-57

Balint JL, Wallace JM, Vukoslavcevic P (1989) The statistical properties of the vorticity field of a two-stream turbulent mixing layer. In: Fernholz HH, Fiedler HE (eds) Proceedings of the 2nd European turbulence conference, Berlin, Germany, September 1989. Springer, Berlin Heidelberg New York, pp 74-78

Batchelor GK, Townsend AA (1949) The nature of turbulent motion at large wave numbers. Proc R Soc Lond Ser A 199(1057):238-255

Bjorkquist DC (2002) Stereoscopic PIV calibration verification. In: Proceedings of the 11th international symposium on application of laser techniques to fluid mechanics, Lisbon, Portugal, July 2002 
Brown LW, Antonia RA, Shah DA (1987) Turbulent energy dissipation in a wake. J Fluid Mech 179:307-326

Brücker C (1997) 3D scanning PIV applied to an air flow in a motored engine using digital high-speed video. Meas Sci Technol 8(12):1480-1492

Clemens NT, Mungal MG (1991) A planar Mie scattering technique for visualizing supersonic mixing flows. Exp Fluids 11:175185

Coudert S, Westerweel J, Fournel T (2000) Comparison between asymmetric and symmetric stereoscopic DPIV system. In: Proceedings of the 10th international symposium on application of laser techniques to fluid mechanics, Lisbon, Portugal, July 2000

Dahm WJA, Su LK, Southerland KB (1992) A scalar imaging velocimetry technique for fully resolved four-dimensional vector velocity field measurements in turbulent flows. Phys Fluids A 4(10):2191-2206

Foss JF (1976) Accuracy and uncertainty of transverse vorticity measurements. Bull Am Phys Soc 21:1237

Hu H, Saga T, Kobayashi T, Taniguchi N, Yasuki M (2001) Dual-plane stereoscopic particle image velocimetry: system set-up and its application on a lobed jet mixing flow. Exp Fluids 31(3):277-293

Kähler CJ, Kompenhans J (1999) Multiple plane stereo PIV: technical realization and fluid-mechanical significance. In: Proceedings of the 3rd international workshop on particle image velocimetry, Santa Barbara, California, September 1999

Kähler CJ, Stanislas M, Kompenhans J (2002) Spatio-temporal flow structure investigation of near-wall turbulence by means of multiplane stereo particle image velocimetry. In: Proceedings of the 11th annual symposium on laser techniques applied to fluid mechanics, Lisbon, Portugal, July 2002

Kasagi N, Nishino K (1991) Probing turbulence with three-dimensional particle tracking velocimetry. Exp Therm Fluid Sci 4:601-613

Kistler AL (1952) The vorticity meter. MS thesis, Johns Hopkins University, Baltimore, Maryland

Kit E, Tsinober A, Dracos T (1993) Velocity gradients in a turbulent jet flow. Appl Sci Res 51:185-190

Kovasznay LSG (1950) Turbulence measurements. In: Landenbuerg RW, Lewis B, Pease RN, Taylor HS (eds) High speed aerodynamics and jet propulsion. Princeton University Press, Princeton, New Jersey, pp 213-285
Lawson NJ, Wu J (1997a) Three-dimensional particle image velocimetry: error analysis of stereoscopic techniques. Meas Sci Technol 8(8):894-900

Lawson NJ, Wu J (1997b) Three-dimensional particle image velocimetry: experimental error analysis of a digital angular stereoscopic system. Meas Sci Technol 8(12):1455-1464

Malik N, Dracos T, Papantoniou D (1993) Particle tracking in threedimensional turbulent flows. Part II: particle tracking. Exp Fluids 15:279-294

Mullin JA, Dahm WJA (2004a) Dual-plane stereo particle image velocimetry measurements of velocity gradient tensor fields in turbulent shear flow. Part I: Assessments. Phys Fluids (submitted)

Mullin JA, Dahm WJA (2004b) Dual-plane stereo particle image velocimetry measurements of velocity gradient tensor fields in turbulent shear flow. Part II: Experimental results. Phys Fluids (submitted)

Prasad AK, Jensen K (1995) Scheimpflug stereocamera for particle image velocimetry in liquid flows. Appl Optics 34(30):7092-7099

Prasad AK, Adrian RJ, Landreth CC, Offutt PW (1992) Effect of resolution on the speed and accuracy of particle image velocimetry interrotation. Exp Fluids 13(2-3):105-116

Raffel M, Willert C, Kompenhans J (1998) Particle image velocimetry: a practical guide. Springer, Berlin Heidelberg New York

Samimy M, Lele SK (1991) Motion of particles with inertia in a compressible free shear layer. Phys Fluids A 3(8):1915-1923

Soloff SM, Adrian RJ, Liu ZC (1997) Distortion compensation for generalized stereoscopic particle image velocimetry. Meas Sci Technol 8(12):1441-1454

Su LK, Dahm WJA (1996a) Scalar imaging velocimetry measurements of the velocity gradient tensor field in turbulent flows. Part I: Assessment of errors. Phys Fluids 8(7):1869-1882

Su LK, Dahm WJA (1996b) Scalar imaging velocimetry measurements of the velocity gradient tensor field in turbulent flows. Part II: Experimental results. Phys Fluids 8(7):1883-1906

Tsinober A, Kit E, Dracos T (1992) Experimental investigation of the field of velocity gradients in turbulent flows. J Fluid Mech 242:169-192

Wieneke B (2003) Stereo-PIV using self-calibration on particle images. In: Proceedings of the 5th international symposium on particle image velocimetry (PIV 2003), Busan, Korea, September 2003

Zhang J, Tao B, Katz J (1997) Turbulent flow measurement in a square duct with hybrid holographic PIV. Exp Fluids 23(5):373-381 